\title{
How should we define a 'good' outcome from encephalitis? A systematic review of the range of outcome measures used in the long-term follow-up of patients with encephalitis
}

\author{
Authors: Harriet Van Den Tooren, ${ }^{\mathrm{A}}$ Ava Easton, ${ }^{\mathrm{B}}$ Cory Hooper, ${ }^{\mathrm{C}}$ Jenny Mullin, ${ }^{\mathrm{D}}$ Jessica Fish, ${ }^{\mathrm{E}}$ Alan Carson, ${ }^{\mathrm{F}}$ \\ Timothy Nicholson, ${ }^{G}$ Tom Solomon ${ }^{H}$ and Benedict D Michael ${ }^{I}$
}

\section{Introduction}

Encephalitis is typically caused by infection or autoimmunity. Most survivors suffer complex neurological and psychiatric sequelae. Standardised outcome measures are needed for accurate interpretation of observational studies and clinical trials. Step one in this process is understanding the strengths and weaknesses of those in use.

\section{Methods}

We performed a systematic literature review searching six databases. One reviewer screened titles and abstracts, and two reviewers determined if shortlisted full-text articles met inclusion criteria. Key data were extracted from these papers and presented as a narrative summary.

Results

Thirty-seven outcome measures were used for 3,133 patients across the $\mathbf{3 5}$ included papers, of which, only one was developed for encephalitis. The outcome measures used in most patients were the Glasgow Outcome Score used in 1,436 (46\%), Barthel Index used in 1,173 (37\%), Euro-QoL-5D used in 1,107 (35\%) and modified Rankin Scale used in 1,034 (33\%).

Authors: ${ }^{A}$ internal medicine trainee, Hull Royal Infirmary, Hull, UK; ${ }^{B}$ chief executive officer, Encephalitis Society, Malton, UK and honorary lecturer, University of Liverpool, Liverpool, UK; ' $\mathrm{PhD}$ student, The Walton Centre NHS Foundation Trust, Liverpool, UK and National Institute for Health Research Health Protection Research Unit in Emerging and Zoonotic Infection, Liverpool, UK; ${ }^{\text {D }}$ linical psychologist, The Walton Centre NHS Foundation Trust, Liverpool, UK; ${ }^{E}$ clinical psychologist and lecturer, University of Glasgow, Glasgow, UK; F consultant neuropsychiatrist and honorary professor, University of Edinburgh, Edinburgh, UK; ${ }^{G}$ clinical lecturer, King's College London, London, UK; ${ }^{H}$ director, National Institute for Health Research Health Protection Research Unit in Emerging and Zoonotic Infection, Liverpool, UK and honorary consultant neurologist, The Walton Centre NHS Foundation Trust, Liverpool, UK; I reader and MRC clinician scientist, National Institute for Health Research Health Protection Research Unit in Emerging and Zoonotic Infection, Liverpool, Liverpool, UK and consultant neurologist, The Walton Centre NHS Foundation Trust, Liverpool, UK

\section{Conclusion}

Most of the 37 measures assessed a single category of sequelae using 5-8-point scales and were not validated for use in encephalitis. Research is needed to develop a composite outcome measure for use in clinical practice and a coreoutcomes set for use in clinical trials. For now, the Liverpool Outcome Score offers a good choice for clinicians.

KEYWORDS: encephalitis, systematic review, outcome

DOI: 10.7861/clinmed.2021-0505

\section{Introduction}

Encephalitis is inflammation of the brain parenchyma that usually presents as an encephalopathy and has an incidence of up to 12.6 per 100,000 per year. ${ }^{1}$ There are a broad range of potential infective and autoimmune aetiologies reflecting demography, geography and comorbidities. ${ }^{2}$

Regardless of aetiology, the majority of survivors suffer long-term sequelae of some form. Around two-thirds of patients fail to make a full recovery even over 2 years after a diagnosis, often reflecting impairments in concentration, behaviour, speech and memory, and seizures, which together can result in significant interpersonal and professional disability. ${ }^{3}$ The combination of both neurological and psychiatric symptoms may explain why so many patients are unable to return to work following encephalitis. ${ }^{4}$ Conversely, although these neurocognitive sequelae are significant, they are potentially missed early in many, as opposed to those occurring in other more common conditions that effect the brain, such as stroke. Because many patients with encephalitis don't have a major obvious physical disability, they are often discharged from hospital with little in the way of plans for rehabilitation. ${ }^{5}$ This lack of early identification risks patients not receiving sufficient early support for their recovery from the brain injury and the appropriate onward referrals for longer-term care not being made. 5,6

Parents of children with encephalitis have changing priorities at different phases of the recovery. ${ }^{7}$ Their priorities after the acute illness focus on the pain their child is experiencing and re-learning of everyday tasks, such as eating, talking and walking. ${ }^{7}$ Later in the child's recovery, parents consider school achievement, participation 
in social activities and wellbeing the most important outcomes. ${ }^{7}$ This mirrors the findings in adult patients following encephalitis, in which the initial challenges they face are symptoms that affect their everyday functioning. ${ }^{4}$ However, in addition to physical and mental health sequelae, persistent challenges relate to changes to employment status and inter-personal relationships. ${ }^{4}$ This was also identified in a narrative assessment of patient outcomes, which identified that it is not only the patients that are affected by the ongoing effects after encephalitis but close family and friends who are often also caregivers. ${ }^{5}$

The Core Outcomes Measures Effectiveness Trials (COMET) Initiative was established to address similar concerns, in particular, ensuring that results of studies can be directly compared: it has been successfully applied to other inflammatory diseases (such as rheumatoid arthritis) and other neurological disorders (such as amyotrophic lateral sclerosis). ${ }^{8,9}$ However, there is currently no core outcome set for encephalitis using the COMET approach. ${ }^{10}$ The heterogeneity in outcome measures used in studies of encephalitis has meant that using meta-analyses to draw conclusions regarding outcomes in these patients is challenging; which is particularly important for clinical trials of the repurposing of existing and novel therapies to reduce cerebral oedema. ${ }^{11}$ Additionally, clinicians have a difficult choice when considering an outcome measure to use for immediate recovery, prognostication or follow-up. Moreover, the importance of integrating patient and family reported outcome measures is increasingly recognised and has resulted in substantive improvements in outcome measures in brain injury due to trauma. ${ }^{12}$ We hypothesise that, due to both the complex neurological, psychiatric, cognitive and physical outcomes and the 'hidden' (ie largely non-physical) nature of these encephalitis sequelae, some of the important outcomes for patients and their relatives are not captured in measures that have been applied routinely.

The first step in the process for developing a dedicated outcome score for patients with encephalitis is to evaluate those existing measures that have been applied in clinical observational studies and trials. This review aims to outline the range of outcome measures that have been used to assess long-term morbidity in patients following encephalitis and see how they compare with features that patients consider important.

\section{Methods}

We searched the Cochrane Library, Web of Science, the National Institute for Health and Care Excellence (NICE) Healthcare Databases Advanced Search (HDAS), Embase, PubMed, MEDLINE and CINAHL from 1990 onwards (search terms in supplementary material S1). A single reviewer screened the titles and abstracts and two reviewers examined the screened full-text articles to determine if they met the inclusion criteria. A single reviewer then extracted relevant data from the included reports (supplementary material S2).

The inclusion criteria were human subjects, the diagnosis of encephalitis (meeting one of the following criteria: aetiology identified with clinical correlation, clinical diagnosis meets consensus statement of the international encephalitis consortium, clinical diagnosis meets the case definition for autoimmune encephalitis or clinical diagnosis meets the International Paediatric Multiple Sclerosis Study Group criteria for the diagnosis of acute disseminated encephalomyelitis (ADEM)), a defined outcome measure was used to follow the patient up, minimum follow-up time of 6 months and full text available in English after

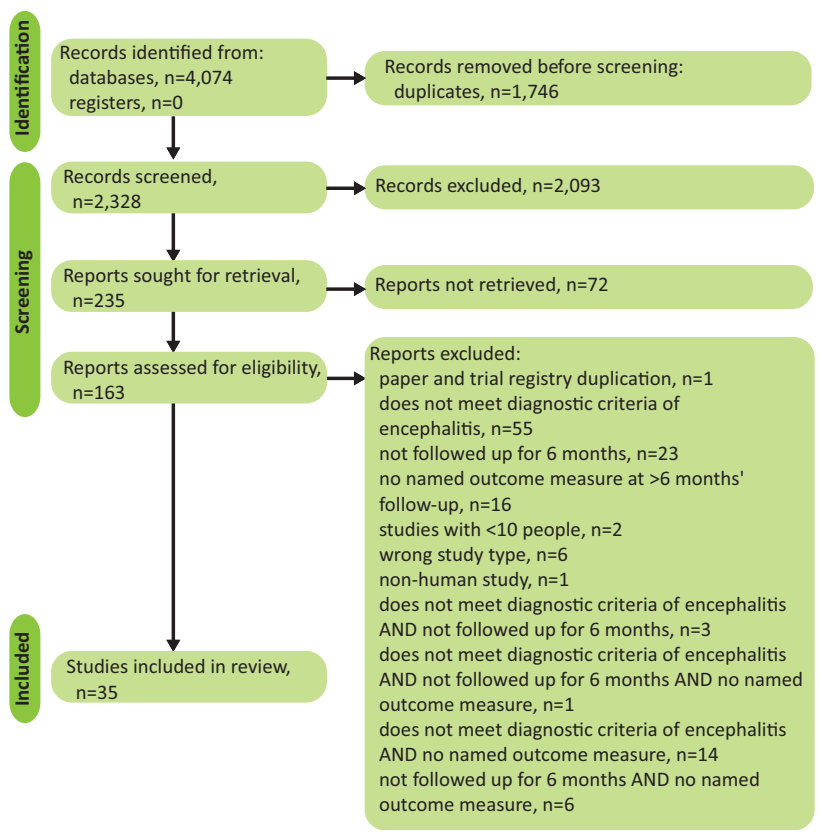

Fig 1. The selection process of articles for review of outcome measures used in the long-term follow-up of patients with encephalitis.

reasonable efforts to translate. ${ }^{13-15}$ Studies were included if they were case series including more than 10 patients, case-control studies, cohort studies and controlled trials.

\section{Results}

In total, 4,074 abstracts were identified, of which, 1,746 were duplicates, therefore, 2,328 abstracts were screened. Two-hundred and thirty-five met the inclusion criteria, of these, 72 were not available in English. One-hundred and sixty-three articles were reviewed in full and 35 met the inclusion criteria, of which, two were protocols (Fig 1).

There were 37 outcome measures used in the 35 included studies that assessed, in total, 3,133 patients (supplementary material S2). Of these outcome measures, 22 were used in only one paper each, while 15 of the outcome measures were used in multiple papers. These outcome measures broadly fall into five categories: physical, cognitive, mood, quality of life and functional outcomes.

The majority of patients were assessed using a 5-to-8-point scale in which patients are rated as either having minimal disability to death. Of the 35 included studies, 18 used the modified Rankin Scale (mRS) and six used the Glasgow Outcome Score (GOS). Of the 3,133 patients, the GOS was used in 1,436 (46\%) and the mRS in $1,034(33 \%)$. Fourteen studies used either the mRS or GOS as its single outcome measure to assess the long-term outcome of 607 (19\%) patients with encephalitis. While the majority of studies assessed patients for physical disability, only 10 assessed cognitive function. A wide range of measures to assess cognitive function were included within the studies, from simple bedside tests usually used to assess patients for dementia to complex neuropsychological batteries that would be carried out by clinical psychologists. Seven studies assessed mood as part of their outcomes, most often testing 
for depression, but two assessed for anxiety and one for general wellbeing. Quality of life was quantified in six of the studies, with one focusing on paediatric quality of life. There were two measures of functional outcome used: the Barthel Index and the Liverpool Outcome Score (LOS). The LOS was the only outcome measure identified that was developed for use in encephalitis, and was used in $370(12 \%)$ patients across six studies. Almost all studies had a limited minimum follow-up of 1 year.

\section{Discussion}

Of the 35 studies identified, there were 37 outcome measures used, which assessed five broad domains. The most assessed category was physical outcomes, in which all of the measures used were developed for other neurological conditions (such as stroke, traumatic brain injury or multiple sclerosis) and most were neither developed nor validated for use in encephalitis. ${ }^{16-18}$ The only outcome measure used within the identified studies that has been developed for use in patients with encephalitis was the LOS, which was not used for $88 \%$ of patients within the included studies. The LOS also includes elements from multiple categories identified within this study, assessing components of physical, cognitive and psychological recovery. ${ }^{19}$

While the outcome measures identified may determine the shortterm physical and mental sequelae of encephalitis appropriately, none addresses the long-term effects of encephalitis, especially on patients' inter-personal relationships, which patients have reported is critically important to their long-term recovery. ${ }^{4}$ Development and validation of an outcome measure that includes the effect of encephalitis on family and wider societal impacts is required. This is critical to both direct clinicians as to what rehabilitation support an individual patient may require and also to direct regional and supra-regional service development and associated quality improvement projects.

As the reliability and validity of an outcome measure can only be determined in relation to a particular population, if these outcome measures are going to be used in future studies of encephalitis, they should be validated in these patients. ${ }^{20}$

The three included protocols had a wider range of outcome measures, spanning more of the five outcome domains with many overlapping outcome assessment methods across the protocols. ${ }^{21-23}$ This suggests the studies will have a more detailed understanding of the sequelae of encephalitis. To solidify this progression, the development of a core outcomes set may be useful. ${ }^{24}$ However, these protocols are for randomised controlled trials and include outcomes measures that would require a long time to administer. In clinical practice and studies with more patient-clinician time constraints, a single composite outcome score may be more useful, which should encompass aspects of all the five outcome domains as well as identifying the effect of illness and recovery on patients' relationships.

\section{Conclusion}

There is a wide variation in the outcome measures used in the assessment of patients following encephalitis, with multiple named assessment tools for a single given problem. Despite the complexity and breadth of sequelae following encephalitis, the majority of patients had outcome measures reported on a 5- or 8-point scale, which would fail to identify effects on mood, cognition or quality of life. Except for the LOS, none of these outcome measures have been developed for assessing outcomes from encephalitis. Encouragingly, recently published protocols are increasing the range of outcome measures used, but there is still significant variation in the choice of these measures. Therefore, research into the sequelae of encephalitis needs to focus on validating the outcome measures in use and determining a core-outcomes set of measures across domains. Throughout this process, identifying outcomes that are important to patients and carers of those who have a lived experience of encephalitis is essential.

For clinicians, the choice of measures that could be used to prognosticate and quantify recovery is vast. While there is no perfect option that encompasses all relevant categories of potentially impaired function, the LOS offers a broad assessment of common sequelae, is easy to administer and, importantly, is validated for use in the encephalitis population.

\section{Key points}

> Sequelae following encephalitis are complex and can affect many aspects of a patient's life including physical capabilities, cognition, mood, quality of life and maintaining relationships.

$>$ For clinicians wanting to use an outcome measure to quantify recovery and direct rehabilitation during immediate and long-term follow-up, the Liverpool Outcome Score offers a good option.

> However, clinicians and researchers need to consider the effect of encephalitis on patients' personal and professional relationships, as this is currently not included on any of the outcome measures in the identified studies; even though it is often critical for patients.

$>$ Work should be undertaken to incorporate and validate patient and clinician reported outcome measures into a composite outcome score of utility in both clinical practice and treatment trials.

\section{Supplementary material}

Additional supplementary material may be found in the online version of this article at www.rcpjournals.org/clinmedicine:

S1 - Terms for database search.

S2 - Summary of included papers.

\section{References}

1 Venkatesan A, Michael BD, Probasco JC, Geocadin RG, Solomon T. Acute encephalitis in immunocompetent adults. Lancet 2019; 393:702-16.

2 Solomon T, Michael BD, Smith PE et al. Management of suspected viral encephalitis in adults - Association of British Neurologists and British Infection Association National Guidelines. Journal of Infection 2012;64:347-73.

3 Mailles A, De Broucker T, Costanzo P et al. Long-term outcome of patients presenting with acute infectious encephalitis of various causes in France. Clinical Infectious Diseases 2012;54:1455-64.

4 Cooper J, Kierans C, Defres S et al. Care beyond the hospital ward: understanding the socio-medical trajectory of herpes simplex virus encephalitis. BMC Health Serv Res 2017;17:646.

5 Easton A. Life after encephalitis. Routledge; 2016

6 Lemon J, Cooper J, Defres S et al. Understanding parental perspectives on outcomes following paediatric encephalitis: A qualitative study. PLOS ONE 2019;14:e0220042.

7 Williamson PR, Altman DG, Bagley $\mathrm{H}$ et al. The COMET Handbook: Version 1.0. Trials 2017;18(Suppl 3):280. 
8 COMET Initiative. Core outcome set for relapsing multiple sclerosis in adults. COMET Initiative, 2020. https://comet-initiative.org/ Studies/Details/1559 [Accessed 19 July 2021].

9 COMET Initiative. Acute encephalitis in children: Which outcomes are important? COMET Initiative, 2014. www.comet-initiative.org/ studies/details/362?result=true

10 Khandaker G, Jung J, Britton PN et al. Long-term outcomes of infective encephalitis in children: a systematic review and meta-analysis. Dev Med Child Neurol 2016;58:1108-15.

11 Ponsford J, Olver J, Ponsford M, Nelms R. Long-term adjustment of families following traumatic brain injury where comprehensive rehabilitation has been provided. Brain Injury 2003;17:453-68.

12 Venkatesan A, Tunkel AR, Bloch KC et al. Case definitions, diagnostic algorithms, and priorities in encephalitis: Consensus statement of the international encephalitis consortium. Clin Infect Dis 2013:57:1114-28.

13 Graus F, Titulaer MJ, Balu R et al. A clinical approach to diagnosis of autoimmune encephalitis. Lancet Neurol 2016;15:391-404.

14 Krupp LB, Tardieu M, Amato MP et al. International Pediatric Multiple Sclerosis Study Group criteria for pediatric multiple sclerosis and immune-mediated central nervous system demyelinating disorders: revisions to the 2007 definitions. Mult Scler 2013;19: 1261-7.

15 Saver JL, Filip B, Hamilton S et al. Improving the reliability of stroke disability grading in clinical trials and clinical practice: The Rankin focused assessment (RFA). Stroke 2010;41:992-5.

16 Mattei TA, Teasdale GM. The Story of the Development and Adoption of the Glasgow Coma Scale: Part I, The Early Years. World Neurosurg 2020;134:311-22.

17 Meyer-Moock S, Feng Y-S, Maeurer M, Dippel F-W, Kohlmann T. Systematic literature review and validity evaluation of the Expanded Disability Status Scale (EDSS) and the Multiple Sclerosis Functional Composite (MSFC) in patients with multiple sclerosis. BMC Neurol 2014;14:58.
18 Lewthwaite P, Begum A, Ooi MH et al. Disability after encephalitis: development and validation of a new outcome score. Bull World Health Organ 2010;88:584-92.

19 Roach KE. Measurement of health Outcomes: Reliability, validity and responsiveness. Journal of Prosthetics and Orthotics 2006; 18:8-12.

20 Davies K. ISRCTN11774734: Does dexamethasone improve outcomes in adults with HSV encephalitis? ISRCTN registry, 2016.

21 Stahl J. EUCTR2016-004835-19-FR: Dexamethasone in herpes simplex virus encephalitis trial. European Union Clinical Trials Register, 2017.

22 Iro MA, Sadarangani M, Absoud M et al. Immunoglobulin in the treatment of encephalitis (IgNiTE): protocol for a multicentre randomised controlled trial. BMJ Open 2016;6:e012356.

23 Prinsen CAC, Vohra S, Rose MR et al. How to select outcome measurement instruments for outcomes included in a 'Core Outcome Set' - a practical guideline. Trials 2016;17:449.

24 DexEnceph. DexEnceph: A study of dexamethasone in adults with Herpes Simplex Virus (HSV) encephalitis. DexEnceph. www. dexenceph.org.uk [Accessed 09 November 2020].

Address for correspondence: Dr Benedict Michael, National Institute for Health Research Health Protection Research

Unit in Emerging and Zoonotic Infection, The Ronald Ross Building, University of Liverpool, 8 West Derby Street, Liverpool L69 7BE, UK.

Email: benedict.michael@liverpool.ac.uk

Twitter:@BenedictNeuro 